International Journal of Pure and Applied Mathematics

Volume 117 No. 2 2017, 335-343

ISSN: 1311-8080 (printed version); ISSN: 1314-3395 (on-line version)

url: http://www.ijpam.eu

doi: 10.12732/ijpam.v117i2.7

\title{
ON MATHEMATICAL MODELING OF THE COMPETITION BETWEEN THE IMMUNE SYSTEM AND VIRAL INFECTIONS
}

\author{
Anka Markovska ${ }^{1}$, Mikhail Kolev ${ }^{2}$, Iveta Nikolova ${ }^{3}$ \\ ${ }^{1}$ Department of Electrical engineering, electronics and automation \\ Faculty of Engineering \\ South-West University "Neofit Rilski" \\ 66, Ivan Mihailov Str., Blagoevgrad, 2700, BULGARIA \\ ${ }^{2,3}$ Department of Mathematics \\ Faculty of Mathematics and Natural Sciences \\ South-West University "Neofit Rilski" \\ 66, Ivan Mihailov Str., Blagoevgrad, 2700, BULGARIA
}

\begin{abstract}
A mathematical model of adaptive immune response to viral infection is formulated as a system of six ordinary differential equations (ODE). The model describes the interactions between virus, uninfected cells, infected cells, and the adaptive immune response represented by antibodies and two subpopulations of cytotoxic T lymphocytes (CTL): CTLprecursors and CTL-effectors. Theorems of existence, uniqueness and non-negativity of solutions are proven. Primary and secondary immune responses against viral infection are investigated by numerical simulations using Matlab.
\end{abstract}

AMS Subject Classification: 65L04, 92C02, 92C06, 92C08

Key Words: numerical simulations, ordinary differential equations, nonlinear dynamics, virus, immune system

\section{Introduction}

Nowadays the development of immunology and medicine is very fast. New treatments and new drugs are being created, which requires long-lasting exper-

$\begin{array}{lr}\text { Received: } & 2017-06-14 \\ \text { Revised: } & 2017-11-15 \\ \text { Published: } & \text { December } 23,2017\end{array}$

(C) 2017 Academic Publications, Ltd. url: www.acadpubl.eu 
iments and clinical trials on animals and humans in order to check the effects of a given medication or a therapy. Sometimes this can lead to undesirable effects in some participants of these experiments or trials. The mathematical modeling approach allows the experimental work to be performed by the use of mathematical models instead of using animals or humans. The results of such experiments become known very quickly. If they are negative there is no need to perform experiments with patients who are desperate due to the applied medical treatment and agree to try anything new, though it is uncertain what will be its results. This requires the creation of new mathematical models in this area and developing and improving existing ones. The models make it possible experimenting on them, not on people.

Many mathematicians work on modeling in the area of medicine. Numerous mathematical models describing the development of cancer diseases with medical treatment and without treatment, the course of viral infections etc. have been created and used for simulation and prognosis.

In this paper we present a mathematical model describing the adaptive immune response to viral infections. The model is a modification of the complicated model presented in [2], which is formulated in terms of kinetic type integro-differential equations. The model presented here is formulated as a system of ordinary differential equations (ODEs) and it is easier for qualitative analysis in comparison with the model in [2]. Another difference between these models is that here we suppose that the concentration of the viruses in the host organism depends not only on the concentration of the infected cells as in [2] but also on the concentration of the free viral particles entering the organism.

The paper is organized as follows. In Section 2 we present a new mathematical model of the competition between immune system and viral infection. Preliminary qualitative analysis of the model is presented. In Section 3 we present results of numerical experiments and give some conclusions and describe our future research plans.

\section{Mathematical model}

We introduce the following notations of the interacting populations considered in our model:

- $x(t)$ - concentration of the susceptible uninfected cells of the target organ;

- $y(t)$ - concentration of the infected cells;

- $v(t)$ - concentration of the free virus particles; 
- $w(t)$ - concentration of antibodies (immunoglobulins) specific for the virus;

- $z(t)$ - concentration of CTL-precursors;

- $r(t)$ - concentration of CTL-effectors.

In our model the dynamics of the population of the susceptible uninfected cells is described by the following equation:

$$
\dot{x}(t)=l-d x(t)-b_{1} x(t) v(t) \text {. }
$$

The meaning of its parameters is the following: parameter $l$ describes the production of uninfected cells; $d$ - the rate of decrease of uninfected cells due to their natural death; $b_{1}$ - the rate of decrease of uninfected cells due to their infection by virus. Parameter $b_{1}$ participates also in the gain term in equation (2) describing the increase of infected cells due to the infection of uninfected cells by viral particles.

The dynamics of the population of the infected cells is described by the following equation:

$$
\dot{y}(t)=b_{1} x(t) v(t)-a y(t)-p y(t) r(t) .
$$

Parameter $a$ characterizes the decrease of concentration of infected cells due to their natural death; $p$ - the rate of decrease of infected cells due to their destruction by CTL-effectors.

The dynamics of the concentration of free virus particles is described by the following equation:

$$
\dot{v}(t)=k y(t) v(t)-q v(t) w(t)-s v(t) .
$$

The meaning of its parameters is the following: $k$ denotes the rate of production of virus particles inside the infected cells; $q$ - the decrease of virus particles due to their neutralization by antibodies; $s$ - the decrease of concentration of the virus particles due to their natural death.

The dynamics of the concentration of antibodies is described by the following equation:

$$
\dot{w}(t)=g v(t) w(t)-h w(t) .
$$

We assume that the production of antibodies depends on the amount of viruses. The meaning of the parameters is the following: $g$ characterizes the production of antibodies; $h$ - the rate of their natural death.

The dynamics of the concentration of cytotoxic T lymphocytes is described by the following equations: 


$$
\begin{gathered}
\dot{z}(t)=c y(t) z(t)-b z(t), \\
\dot{r}(t)=m y(t) z(t)-n r(t) .
\end{gathered}
$$

Parameters $c$ and $m$ denote the rates of production of precursor (CTLp) and effector (CTLe) cytotoxic T lymphocytes, respectively. Note that the rates of production of both subpopulations are proportional not only to the concentration of the infected cells, but also to the concentration of the precursor CTL. Parameters $b$ and $n$ denote the rate of natural death of CTLp and CTLe, respectively. Since the precursor CTL are responsible for the establishment of CTL memory, we suppose that $c<<n$ (CTLp are assumed to live much longer than CTLe).

We suppose that the parameters of the model system (1) - (6) are nonnegative constants and parameter $l$ is positive. We look for solution such that the unknown functions are continuously differentiable with non-negative initial conditions.

Now we formulate two theorems concerning the properties of the solutions to initial value problem related to the system (1) - (6). The first theorem treats the non-negativity of the solutions.

Theorem 1. If the system (1) - (6) with initial conditions $x\left(t_{0}\right)=x_{0}>0$, $y\left(t_{0}\right)=y_{0} \geq 0, v\left(t_{0}\right)=v_{0} \geq 0, w\left(t_{0}\right)=w_{0} \geq 0, z\left(t_{0}\right)=z_{0} \geq 0, r\left(t_{0}\right)=r_{0} \geq 0$, possesses a solution, then this solution is non-negative for every $t \geq t_{0}$.

Proof. Consider equation (1). Let us assume that there exist values of $t>t_{0}$ such that $x(t)<0$. From the initial condition $x\left(t_{0}\right)>0$ and the continuity of the function $x(t)$ it follows that there exists an instant in time $t_{1}>t_{0}$ at which $x(t)$ changes its sign, $x\left(t_{1}\right)=0$ and let $t_{1}$ be the smallest value of $t$ for which $x\left(t_{1}\right)=0$. From here we would have $\dot{x}\left(t_{1}\right) \leq 0$. This would be a contradiction with equation (1) from which follows that

$$
\dot{x}\left(t_{1}\right)=l-d x\left(t_{1}\right)-b_{1} x\left(t_{1}\right) v\left(t_{1}\right)=l>0 .
$$

Therefore the assumption about the possible negativity of $x(t)$ is incorrect.

From equation (3) it follows that:

$$
v(t)=v\left(t_{0}\right) e^{\int_{t_{0}}^{t}[k y(u)-q w(u)-s] d u} \geq 0 \text { for } t \geq t_{0} .
$$

From equation (4) it follows that:

$$
w(t)=w\left(t_{0}\right) e^{\int_{t_{0}}^{t}[g v(u)-h] d u} \geq 0 \text { for } t \geq t_{0} .
$$


From the non-negativity of $x(t)$ and $v(t)$ the non-negativity of $y(t)$ follows the non-negativity of $x(t)$ and $v(t)$ the non-negativity of $y(t)$ follows, since from equation (2) we obtain:

$$
y(t)=e^{-\int_{t_{0}}^{t}[p r(u)+a] d u}\left[y(0)+\int_{t_{0}}^{t} b_{1} x(u) v(u) e^{\int_{t_{0}}^{t}\left[p r\left(u_{1}\right)+a\right] d u_{1}} d u\right] \geq 0
$$

for $t \geq t_{0}$, because $x(t) \geq 0$ and $v(t) \geq 0$.

From equation (5) the non-negativity of $z(t)$ follows, since

$$
z(t)=z\left(t_{0}\right) e^{\int_{t_{0}}^{t}[c y(u)-b] d u} \geq 0 \text { for } t \geq t_{0} .
$$

Similarly, from equation (6) the non-negativity of $r(t)$ follows, since

$$
r(t)=e^{\int_{t_{0}}^{t} n d u}\left[r(0)+\int_{t_{0}}^{t} m y(u) z(u) e^{\int_{t_{0}}^{t} n d u_{1}}\right] \geq 0 \text { for } t \geq t_{0},
$$

because $y(t) \geq 0$ and $z(t) \geq 0$.

The second theorem is related to the existence and uniqueness of the solution to model system (1) - (6).

Theorem 2. For every $T>t_{0}$, in the interval $\left[t_{0}, T\right]$ there exists an unique continuously differentiable solution to the system (1) - (6) with initial conditions $x\left(t_{0}\right)=x_{0}>0, y\left(t_{0}\right)=y_{0} \geq 0, v\left(t_{0}\right)=v_{0} \geq 0, w\left(t_{0}\right)=w_{0} \geq 0$, $z\left(t_{0}\right)=z_{0} \geq 0, r\left(t_{0}\right)=r_{0} \geq 0$.

Proof. The local existence of the solution follows from the continuity of the right-hand sides [1]. The uniqueness of the solution follows from the continuity of the partial derivatives of the right-hand sides with respect to the unknown functions [1].

In order to prove the global existence we need to find a priori bounds of the solution. It can be shown that the functions $x(t), y(t), v(t), w(t), z(t)$ and $r(t)$ are bounded on $\left[t_{0} ; T\right]$, therefore they attain their maximal and minimal values on $\left[t_{0} ; T\right]$. Let us denote their maximal values with $X, Y, V, W, Z$ and $R$ respectively.

The following a priory bounds can be established for the solution on $\left[t_{0}, T\right]$. First, we have

$$
\dot{x}(t)=l-d x(t)-b_{1} x(t) y(t) \leq l,
$$

therefore 


$$
x(t) \leq x\left(t_{0}\right)+l T=X,
$$

which means that $x(t)$ is bounded from above on the interval $\left[t_{0}, T\right]$. After summing eqs. (1) and (2) we obtain:

$$
\frac{d(x(t)+y(t))}{d t} \leq l
$$

and therefore

$$
x(t)+y(t) \leq l . T+x\left(t_{0}\right)+y\left(t_{0}\right) .
$$

From here it follows that $y(t)$ is also bounded from above on the interval $\left[t_{0}, T\right]$. Let $y(t) \leq Y, t \in\left[t_{0}, T\right]$.

From eq. (3) it follows that

$$
\dot{v}(t)=k \cdot y(t) \cdot v(t)-q \cdot v(t) \cdot w(t)-s \cdot v(t) \leq k \cdot Y \cdot v(t),
$$

which leads to:

$$
v(t) \leq \exp \left[\ln \left(v\left(t_{0}\right)+k \cdot Y \cdot\left(T-t_{0}\right)\right)\right],
$$

and therefore $v(t)$ is also bounded from above on the interval $\left[t_{0}, T\right], v(t) \leq$ $V$, where

$$
V=\exp \left[\ln \left(v\left(t_{0}\right)+k \cdot Y \cdot\left(T-t_{0}\right)\right)\right] .
$$

From eq. (4) it follows that

$$
\dot{w}(t)=g v(t) w(t)-h w(t) \leq g . V \cdot w(t) .
$$

From eq. (5) it follows that

$$
\dot{z}(t)=c y(t) z(t)-b z(t) \leq c . Y . z(t) .
$$

From eq. (6) it follows that

$$
\dot{r}(t)=m y(t) z(t)-n r(t) \leq m . Y . z(t) .
$$

Therefore the system (1) - (6) behaves not worse than a linear system. Hence a global solution on $\left[t_{0} ; T\right]$ exists, which is unique. 


\section{Numerical experiments and discussion}

The Cauchy problem (1)-(6) consisting of six nonlinear ODE is solved numerically. The system is solved by using the code ode15s from the Matlab ODE suite with $\operatorname{RelTol}=10^{-3}$ and $A b s T o l=10^{-4}$. ode15s is a multistep solver using numerical differential formulae (see, e.g. [3]).

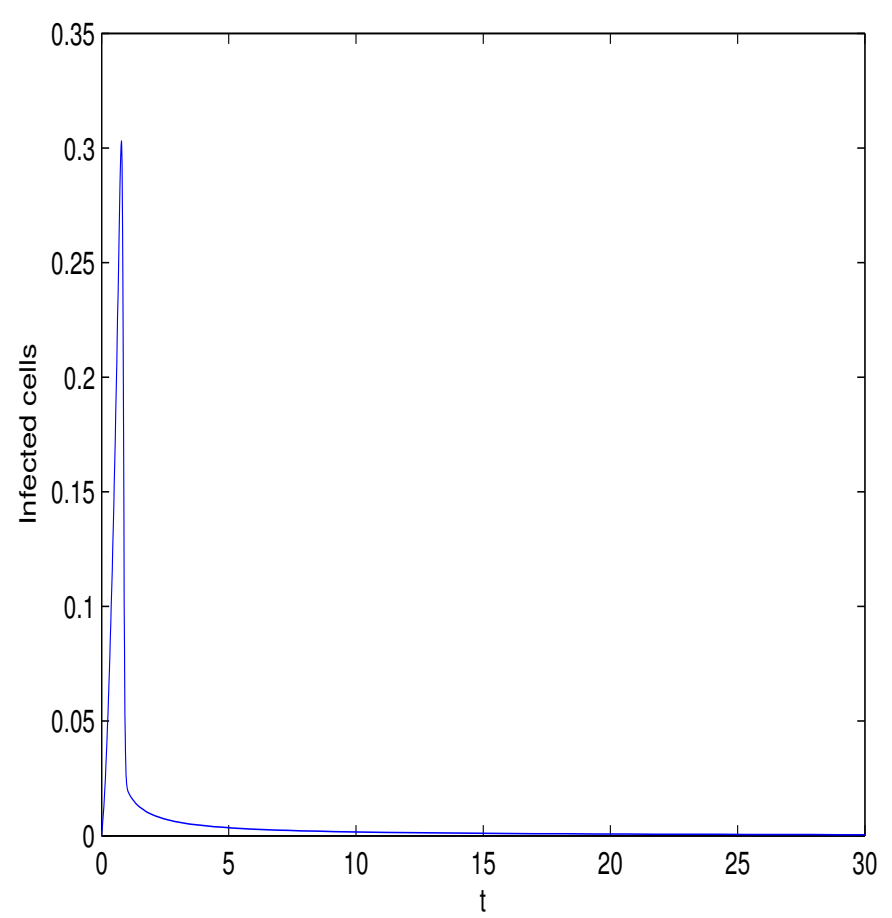

Figure 1: Concentration of infected cells without vaccination

As initial conditions we assume the presence of uninfected cells, free virus particles, antibodies, CTLp, and the absence of infected cells and CTLe, setting :

$$
t_{0}=0, x(0)=1, y(0)=0, \quad v(0)=0.5, \quad w(0)=0.1, \quad r(0)=0 .
$$

We simulate two cases of disease treatment: without vaccination, setting $z(0)=0.001$, and with vaccination at $t=0, z(0)=0.1$. In the second case the 
concentration of CTLp is higher due to the vaccination. The dynamics of the infected cells in the first case is shown in Fig. 1 and in the second case - in Fig. 2.

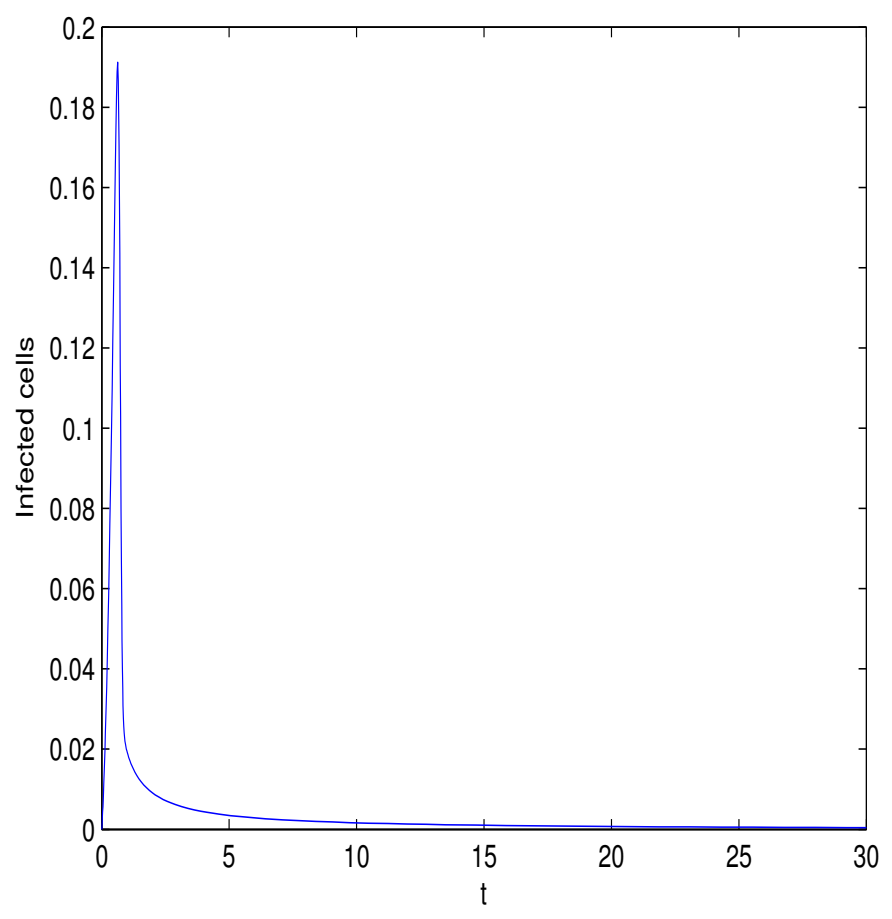

Figure 2: Concentration of infected cells after vaccination

The obtained results show that providing vaccination can lead to fast decrease in the concentration of infected cells and enhance the immune response.

In our future work we plan to apply the model to specific clinical data. 


\section{References}

[1] P. Hartman, Ordinary Differential Equations, John Wiley and Sons, New York-LondonSydney (1964).

[2] M. Kolev, A. Korpusik, A. Markovska, Adaptive immunity and CTL differentiation - a kinetic modeling approach, Mathematics in Engineering, Science and Aerospace (MESA), 3 (2012), 285-293.

[3] L. Shampine, M. Reichelt, The Matlab ODE suite. SIAM J. Sci. Comput., 18 (1997), $1-22$. 
